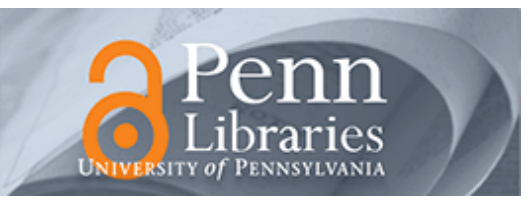

University of Pennsylvania

ScholarlyCommons

\title{
Novel Electrode Configurations in Dual-Layer Stacked and Switchable AIN Contour-Mode Resonators for Low Impedance Filter Termination and Reduced Insertion Loss
}

\author{
Chengjie Zuo \\ University of Pennsylvania, chengjiezuo@hotmail.com \\ Nipun Sinha \\ University of Pennsylvania, nipun@seas.upenn.edu \\ Gianluca Piazza \\ University of Pennsylvania, piazza@seas.upenn.edu
}

Follow this and additional works at: https://repository.upenn.edu/ese_papers

Part of the Electrical and Electronics Commons

\section{Recommended Citation}

Chengjie Zuo, Nipun Sinha, and Gianluca Piazza, "Novel Electrode Configurations in Dual-Layer Stacked and Switchable AIN Contour-Mode Resonators for Low Impedance Filter Termination and Reduced Insertion Loss", 23rd IEEE International Conference on Micro Electro Mechanical Systems (MEMS 2010), 719-722. January 2010.

Suggested Citation:

Chengjie Zuo, Nipun Sinha, and Gianluca Piazza. "Novel Electrode Configurations in Dual-Layer Stacked and Switchable AIN Contour-Mode Resonators for Low Impedance Filter Termination and Reduced Insertion Loss" 23rd IEEE International Conference on Micro Electro Mechanical Systems (MEMS 2010) (2010): 719-722. (C2010 IEEE. Personal use of this material is permitted. However, permission to reprint/republish this material for advertising or promotional purposes or for creating new collective works for resale or redistribution to servers or lists, or to reuse any copyrighted component of this work in other works must be obtained from the IEEE.

This paper is posted at ScholarlyCommons. https://repository.upenn.edu/ese_papers/543

For more information, please contact repository@pobox.upenn.edu. 


\title{
Novel Electrode Configurations in Dual-Layer Stacked and Switchable AIN Contour-Mode Resonators for Low Impedance Filter Termination and Reduced Insertion Loss
}

\author{
Abstract \\ This paper reports, for the first time, on the design and demonstration of two novel electrode \\ configurations in dual-layer stacked Aluminum Nitride (AIN) piezoelectric contour-mode resonators to \\ obtain low filter termination resistance (down to $300 \Omega$, which also results in better filter out-of-band \\ rejection) and reduced insertion loss (IL as low as $1.6 \mathrm{~dB}$ ) in multi-frequency $(100 \mathrm{MHz}-1 \mathrm{GHz}$ ) AIN \\ MEMS filters. The microfabrication process is fully compatible with the previously demonstrated AIN RF \\ MEMS switches, which makes it possible to design and integrate multi-frequency switchable filter banks \\ on a single chip. \\ Disciplines \\ Electrical and Electronics \\ Comments \\ Suggested Citation: \\ Chengjie Zuo, Nipun Sinha, and Gianluca Piazza. "Novel Electrode Configurations in Dual-Layer Stacked \\ and Switchable AIN Contour-Mode Resonators for Low Impedance Filter Termination and Reduced \\ Insertion Loss" 23rd IEEE International Conference on Micro Electro Mechanical Systems (MEMS 2010) \\ (2010): 719-722. \\ C2010 IEEE. Personal use of this material is permitted. However, permission to reprint/republish this \\ material for advertising or promotional purposes or for creating new collective works for resale or \\ redistribution to servers or lists, or to reuse any copyrighted component of this work in other works must \\ be obtained from the IEEE.
}




\title{
NOVEL ELECTRODE CONFIGURATIONS IN DUAL-LAYER STACKED AND SWITCHABLE ALN CONTOUR-MODE RESONATORS FOR LOW IMPEDANCE FILTER TERMINATION AND REDUCED INSERTION LOSS
}

\author{
Chengjie Zuo, Nipun Sinha, and Gianluca Piazza \\ Penn Micro and Nano Systems (PMaNS) Laboratory, University of Pennsylvania \\ Philadelphia, PA, 19104, USA
}

\begin{abstract}
This paper reports, for the first time, on the design and demonstration of two novel electrode configurations in dual-layer stacked Aluminum Nitride (AlN) piezoelectric contour-mode resonators to obtain low filter termination resistance (down to $300 \Omega$, which also results in better filter out-of-band rejection) and reduced insertion loss (IL as low as $1.6 \mathrm{~dB})$ in multi-frequency $(100 \mathrm{MHz}-1 \mathrm{GHz})$ AlN MEMS filters. The microfabrication process is fully compatible with the previously demonstrated AlN RF MEMS switches, which makes it possible to design and integrate multi-frequency switchable filter banks on a single chip.
\end{abstract}

\section{INTRODUCTION AND MOTIVATION}

A promising solution for single-chip multiple frequency reconfigurable wireless communications is the use of narrow-band filter banks that span the entire frequency range of interest and realize direct channel selection at the radio frequency (RF) stage [1-3]. Emerging resonator technologies based on microelectromechanical systems (MEMS) can provide high quality factor, single-chip multi-frequency operation, and CMOS compatibility [1, 4]. Either using electrostatic [5] or piezoelectric [6] MEMS resonators, band-pass filters have been previously demonstrated with narrow bandwidth $(<1 \%)$ and low insertion loss $(\sim 4 \mathrm{~dB})$. However, for the ultimate goal of channel selection, more stringent specifications need to be met.

First of all, the fractional bandwidth, defined as the filter $3 \mathrm{~dB}$ bandwidth divided by the center frequency, has to be small enough to really select the desired RF signal channel with good rejection of adjacent channels. The detailed specifications for bandwidth may vary depending on the particular wireless communication standard. For example, the channel width of the IEEE 802.11a wireless standard is defined as $16.6 \mathrm{MHz}$ at $5 \mathrm{GHz}$, and, correspondingly, the channel bandwidth is calculated as $0.3 \%$ [7]. On the other hand, it is also preferable to pursue narrower channel bandwidth for more efficient electromagnetic spectral utilization and higher signal-to-noise ratio [1]. In terms of providing small bandwidth $<1 \%$, various research groups $[1,5,8]$ have already demonstrated that MEMS filters are perfectly suitable. However, these demonstrations have shown limited out-of-band rejection $(\sim 36 \mathrm{~dB})$ and only the use of self-coupling technique in the piezoelectric Aluminum Nitride (AlN) contour-mode MEMS resonator technology has shown rejection in excess of $60 \mathrm{~dB}[6]$.
Second, extremely low insertion loss is very critical for direct channel selection at the RF stage. For traditional RF architectures, channel selection is usually performed at the baseband frequency after the low noise amplifier (LNA) and mixer stages, so as to relax the high $Q$ requirements on the filters. It is also possible to place the channel-select filter before the mixer, but this is done at the cost of increasing the RF signal gain (i.e., power consumption) of the LNA [7]. In all these cases, insertion loss is not a significant issue, since the signal is already amplified before it goes through the channel selector. However, if direct RF channel selection and filtering are carried out before the LNA stage, the insertion loss of the channel-select filter becomes the most critical parameter in the whole receiver chain, since solely $1 \mathrm{~dB}$ degradation of the RF signal may result in extra burden on the subsequent stages (LNA, mixer, local oscillator, etc.) in terms of gain, noise figure, and linearity. In order to satisfy the same specification, orders of magnitude more DC power consumption or chip area occupation may be required to account for the RF power loss in the first stage. Therefore, the insertion loss of an RF channel-select filter has to be as small as possible. For MEMS filters with narrow bandwidth, an insertion loss of $\sim 4 \mathrm{~dB}$ has been previously demonstrated $[1,5-6,8]$, but an ultimate low loss lower than $3 \mathrm{~dB}$ for multiple frequency operation from $100 \mathrm{MHz}$ up to several $\mathrm{GHz}$ on a single chip is desired. This is still a significant challenge for narrow band filters based on MEMS resonators. Since insertion loss is generally inversely proportional to the $k_{t}^{2} Q$ product and $k_{t}^{2}$ usually needs to be small for the narrow bandwidth, then the required low insertion loss would have to rely on an extremely high $Q$, which is very hard to design or control, especially over such a wide frequency range.

Finally, the RF channel-select filter should also serve as a bridge to connect the antenna with the integrated circuits (ICs). Antennas generally have a characteristic impedance of $50 \Omega$, while transceiver ICs usually work at higher impedance. Therefore it is desirable for the channel-select filter to have low device impedance ranging from $50 \Omega$ to several $\mathrm{k} \Omega$, so that impedance matching is realized through the filter itself and extra power loss in external matching networks is avoided. Due to their small size, MEMS devices usually have much higher impedance than conventional discrete electronic components, and the termination resistance has been recently reduced to only a few $\mathrm{k} \Omega$ for electrostatic MEMS filters [1]. As for piezoelectric AlN contour-mode MEMS resonators, a well-recognized advantage is the lower impedance and a $50 \Omega$ filter termination can be ultimately realized by 
AIN Input Pt Electrodes $\quad$ Output Pt Electrodes

Floating Pt Electrodes
Grounded Pt Electrodes (a) $\mathrm{SL}$

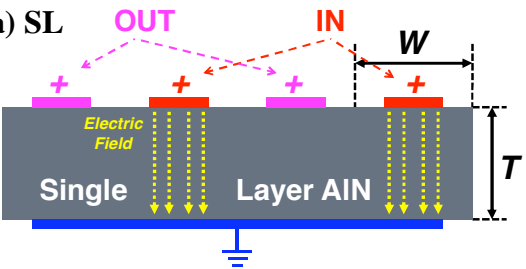

Conventional Single-Layer AIN Filter
Impedance

Reduction

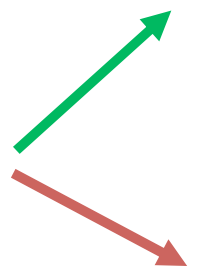

More Effective

Excitation

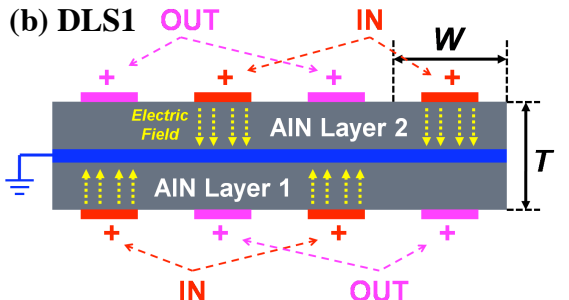

(c) DLS2

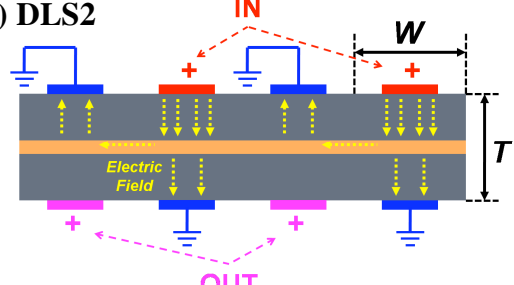

Figure 1: Cross-sectional schematics of different electrode configurations for two-port resonators and filter synthesis in a dual-layer AlN MEMS process: (a) SL, (b) DLS1, and (c) DLS2. The electric field distributions in the novel dual-layer solutions are schematic representations used to illustrate the operating principle of the devices.

properly designing the transducer capacitance.

In order to address some of the aforementioned issues for the synthesis of channel-select filters, two novel electrode configurations in dual-layer stacked (DLS) piezoelectric AlN contour-mode MEMS resonators have been designed and implemented. This work further lowers the filter termination resistance (as low as $300 \Omega$, which also results in a higher out-of-band rejection up to $75 \mathrm{~dB}$ at $99 \mathrm{MHz}$ ) of self-coupled narrowband filters over a wide frequency range from $100 \mathrm{MHz}$ to $1 \mathrm{GHz}$. Another DLS electrode configuration is also shown to attain low insertion loss of $1.6 \mathrm{~dB}$ at $250 \mathrm{MHz}$ and $4.0 \mathrm{~dB}$ at $1 \mathrm{GHz}$. The fabrication process used to make the DLS filters is fully compatible with the previously demonstrated piezoelectric AIN RF MEMS switches [9], which makes it possible to integrate multi-frequency switchable filter banks on a single chip. As a proof of concept, one of the new DLS low frequency (99 MHz) filters was effectively turned on and off by a piezoelectric AlN RF MEMS switch fabricated next to it on the same chip.

\section{RESONATOR AND FILTER DESIGN}

Two novel electrode configurations (named as DLS1 and DLS2 in this paper) for two-port piezoelectric AlN contour-mode resonators in a dual-layer stacked (DLS) AlN MEMS process are explored in this work. Fig. 1 compares the cross-sectional schematics of the two DLS configurations with respect to the conventional single layer (SL) version. The use of two layers of stacked AlN thin films (600 nm each) enables freedom in the routing of electric fields within the resonant body without adding more tethers (which introduce anchor loss) or increasing the device form factor. The higher order filters are synthesized by directly cascading these two-port AlN MEMS resonators according to a self-coupling technique previously described in [6].

As shown in Fig. 1 (b) for DLS1, the same volume of the AlN plate forming a SL device is divided into two and an electrically grounded $\mathrm{Pt}$ electrode layer is inserted in between the two AlN layers so that both the input and output transducer capacitance is increased by 4 times. For optimum performance, piezoelectric filters need to be terminated by a proper termination resistance, which is generally equal to the magnitude of the corresponding input or output transducer impedance. Consequently the termination resistance of DLS1 filters is reduced to a large extent (as low as $300 \Omega$ ) thanks to the 4 times increase in the transducer capacitance. At the same time, the use of a $4 \mathrm{X}$ lower filter termination resistance also results in $12 \mathrm{~dB}$ improvement in the out-of-band rejection for the same level of feed-through capacitance. By reducing the film thickness and further increasing the number of transducer fingers [10], the filter termination can be easily lowered to $50 \Omega$, so as to facilitate the direct interface between these filters and conventional RF components.

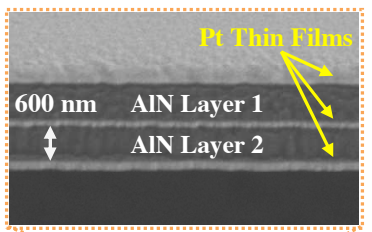

(a)

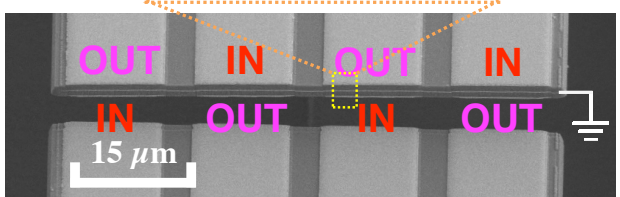

(b)

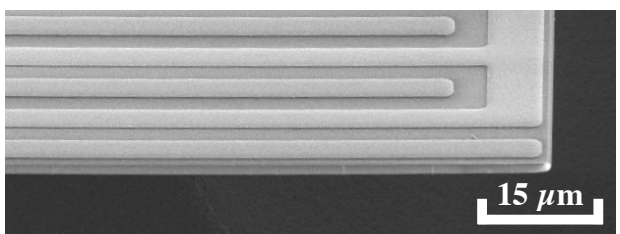

Figure 2: (a) SEM pictures of the fabricated devices showing two cross-sectional views of the dual-layer stacked AlN two-port resonators. The top figure shows the layers forming the resonator. The bottom part shows a 
cut-out view of the electrode configuration for DLS1. (b) Results of AlN sidewall etching after process optimization with photoresist as a mask at low temperature $\left(12{ }^{\circ} \mathrm{C}\right)$. This step is also responsible for improved insertion loss.

In the DLS2 configuration illustrated in Fig. 1 (c), a floating Pt electrode is placed in between the two AlN piezoelectric layers instead of the grounded electrode. Due to this special electrode routing, three quarters of the AlN plate is excited by the input RF signal instead of only half the volume as for conventional two-port resonators. This corresponds to a more effective coupling into the desired mode of vibration.

\section{DEVICE FABRICATION}

The fabrication process uses 5 masks and coincides with the first 5 steps that were used to make AIN RF MEMS switches $[9,11]$. In this case, a new method for the direct etching of AlN at low temperature $\left(12{ }^{\circ} \mathrm{C}\right)$ with photoresist as a mask, instead of the conventional $\mathrm{SiO}_{2}$ hard mask, has been adopted to improve the etching profile and therefore the resonator edges (Fig. 2). In this way, the etching step for the definition of the AlN resonator body has been greatly simplified and the surface cleanliness of the wafer has been improved to a large extent.

\section{EXPERIMENTAL RESULTS}

The fabricated filters were directly probed at ambient conditions in a Desert Cryogenics probe station and measured by an Agilent N5230A network analyzer after SOLT calibration. The measurement data are listed in Table I. The results for conventional SL filters on the same chip are also given for comparison.

TABLE I. EXPERIMENTAL RESULTS OF SL AND DLS FILTERS

\begin{tabular}{|c|c|c|c|c|c|c|c|c|}
\hline Filter & $\begin{array}{c}\boldsymbol{f}_{\boldsymbol{c}} \\
{[\mathbf{M H z}]}\end{array}$ & $\begin{array}{c}\boldsymbol{I L} \\
{[\mathbf{d B}]}\end{array}$ & $\boldsymbol{F B W _ { 3 \mathrm { dB } }}$ & $\begin{array}{c}\boldsymbol{R}_{\boldsymbol{T}} \\
{[\mathbf{\Omega}]}\end{array}$ & $\boldsymbol{O r d e r}$ & $\boldsymbol{R} \boldsymbol{e j}$ & $\boldsymbol{S F}_{\mathbf{2 0 d B}}$ & $\boldsymbol{S F}_{\text {50dB }}$ \\
\hline SL & 251 & 2.3 & $0.53 \%$ & 1200 & 3 & 60 & 2.0 & 5.3 \\
\hline SL & 639 & 4.8 & $0.22 \%$ & 800 & 3 & 55 & 2.3 & 7.8 \\
\hline SL & 1276 & 10.9 & $0.12 \%$ & 700 & 3 & 49 & 6.3 & - \\
\hline DLS1 & 99 & 3.9 & $0.35 \%$ & 400 & 3 & 75 & 2.1 & 5.8 \\
\hline DLS1 & 250 & 3.9 & $0.44 \%$ & 300 & 3 & 70 & 1.9 & 6.9 \\
\hline DLS1 & 1043 & 11.2 & $0.34 \%$ & 300 & 3 & 55 & 3.7 & 19.1 \\
\hline DLS2 & 250 & 1.6 & $0.48 \%$ & 1400 & 2 & 20 & 3.1 & - \\
\hline DLS2 & 707 & 3.0 & $0.30 \%$ & 600 & 2 & 20 & 9.6 & - \\
\hline DLS2 & 1035 & 4.0 & $0.40 \%$ & 700 & 2 & 20 & 8.6 & - \\
\hline
\end{tabular}

SL: single-layer AIN filters; DLS: dual-layer stacked AlN filters; $f_{c}$ : filter center frequency; $I L$ : insertion loss; $F B W_{3 \mathrm{~dB}}: 3 \mathrm{~dB}$ fractional bandwidth; $R_{T}$ : termination resistance; Order: filter order; $R e j$ : out-of-band rejection; $S F$ : shape factor;

For the $251 \mathrm{MHz}$ SL AlN filter, a reduced insertion loss of $2.3 \mathrm{~dB}$ from $4.2 \mathrm{~dB}$ has been observed when compared to a previous demonstration with the same design [6]. This improved performance can be primarily attributed to the increased electromechanical coupling coefficient $\left(k_{t}^{2}\right)$ from $1.5 \%$ to $1.9 \%$ at a similar $Q$. As for the DLS1 filters, clearly lower filter termination resistance $(\sim 300 \Omega$, as highlighted in Table I) is obtained when compared with the SL counterparts. The insertion loss, instead, shows some deterioration. The authors believe that this is mainly caused by the additional fabrication complexity. As an example, the response of the $250 \mathrm{MHz}$ DLS1 filter is given in Fig. 3. Compared with the SL filter at a similar frequency, this filter requires approximately one quarter of the termination resistance and also exhibits $10 \mathrm{~dB}$ improvement in the out-of-band rejection. The relatively large spurious peak shown in Fig. 3 is due to the specific aspect ratio of the resonator and is worsened by the $1 \mu \mathrm{m}$ misalignment during the fabrication process.

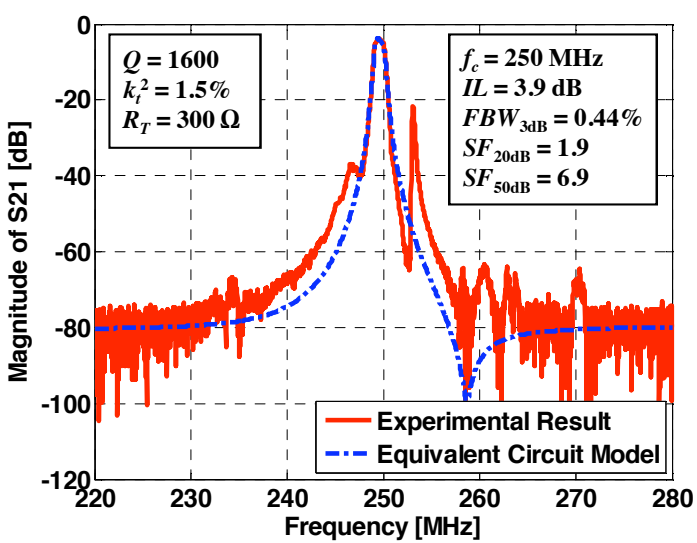

Figure 3: Response of the $250 \mathrm{MHz}$ DLS1 filter showing a low termination resistance of $300 \Omega$. Note: the $Q$ and $k_{t}^{2}$ values listed here refer to the quality factor and the electromechanical coupling coefficient of the composing resonators, as is the same for Fig. 4.

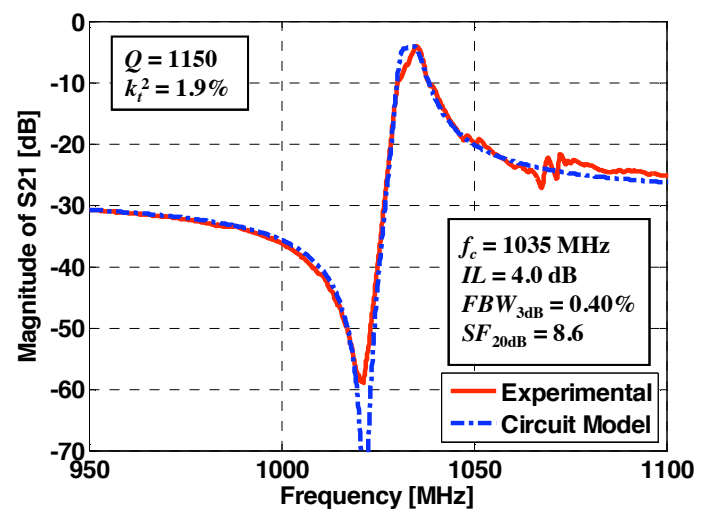

Figure 4: Response of the $1035 \mathrm{MHz}$ DLS2 filter showing low IL of $4.0 \mathrm{~dB}$.

Despite the misalignment, the DLS2 filters show high $k_{t}^{2} Q$ product in the composing resonators, as can be seen from the low insertion loss obtained over a wide frequency range: $1.6 \mathrm{~dB}$ at $250 \mathrm{MHz}$ and $4.0 \mathrm{~dB}$ at $1.035 \mathrm{GHz}$. By comparing the different electrode configurations at the same frequency, the lowered insertion loss is primarily due to the increased $Q$ in the DLS2 configuration. The $250 \mathrm{MHz}$ DLS2 resonator has a $Q$ of 3000 and a $k_{t}^{2}$ of $1.6 \%$; the $1.035 \mathrm{GHz}$ DLS2 resonator has a $Q$ of 1150 and a $k_{t}^{2}$ of $1.9 \%$ (Fig. 4). The authors believe that the better performance is likely due to the improved efficiency in which the device is forced into vibrations and depends on the specific electrode arrangement. The dip before the 
main filter passband and the poor out-of-band rejection of DLS2 filters are caused by the intrinsic feed-through capacitance existing between the input and output ports, as shown in Fig. 5.

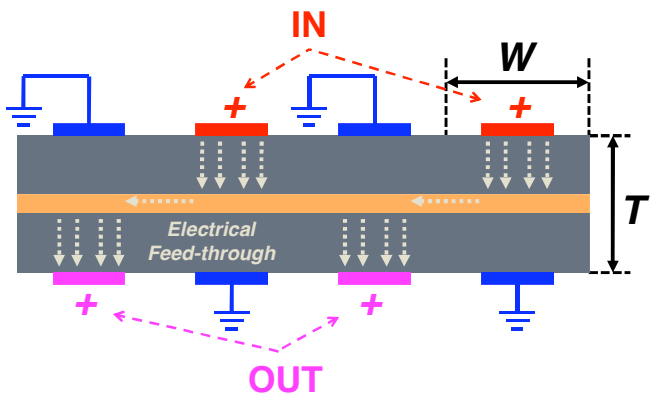

Figure 5: Cross-sectional schematic of DLS2 filters illustrating intrinsic feed-through capacitance existing between the input and output ports.

As a proof of concept for the single-chip switchable filter banks, one of the DLS1 low frequency ( $99 \mathrm{MHz}$ ) filters was integrated with a piezoelectric AlN RF MEMS switch and effectively turned on and off by the switch, as shown in Fig. 6.

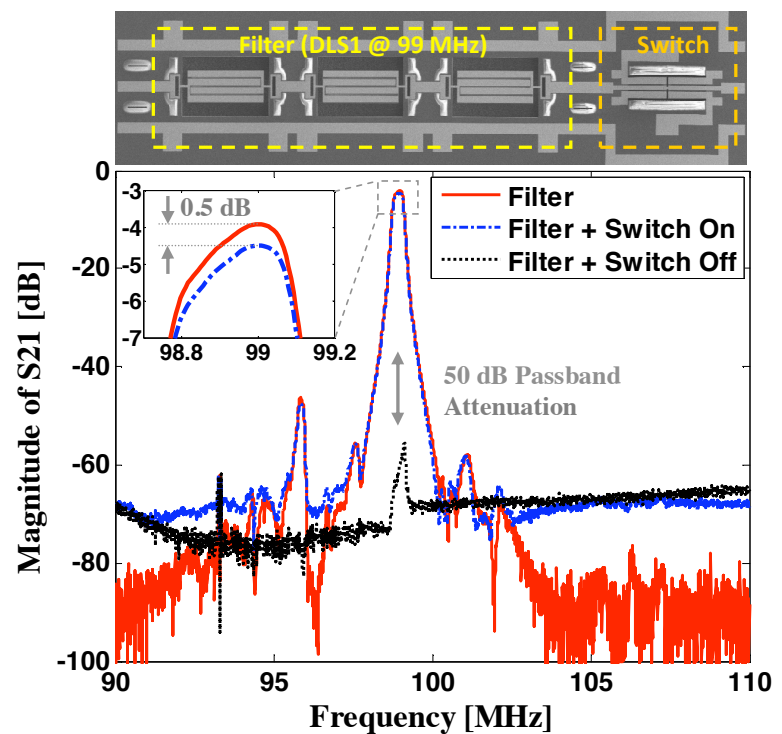

Figure 6: SEM picture and experimental results showing a high-performance dual-layer AlN filter effectively turned on and off by an AlN RF MEMS switch integrated on the same chip. The switch introduces low loss and enables a single-chip switchable filter bank solution.

\section{CONCLUSIONS}

Single-chip multi-frequency channel-select filters have been designed, fabricated, and tested based on dual-layer stacked AlN contour-mode MEMS resonators. The dual layer stacking of two piezoelectric AIN thin films enables more freedom in the routing of electric field within the resonant structure without adding more tethers (which introduce anchor loss) or increasing the device size. Two novel electrode configurations have been proposed to further reduce the filter termination resistance (which results in improved out-of-band rejection for the same feed-through capacitance) and insertion loss. The filter termination resistance has been reduced to $300 \Omega$, and low insertion loss (1.6 dB at $250 \mathrm{MHz}$ and $4.0 \mathrm{~dB}$ at $1 \mathrm{GHz})$ has been achieved in $2^{\text {nd }}$ order narrow-band band-pass AIN MEMS filters operating in ambient conditions. A prototype of a single-chip integrated switchable filter at $99 \mathrm{MHz}$ has been implemented to show the possibility to realize multi-frequency reconfigurable filter banks and wireless front-ends. In future work, we plan to combine the features of low termination and low loss in a single device configuration by further optimizing this DLS AlN technology.

\section{REFERENCES}

[1] C. T.-C. Nguyen, "MEMS Technology for Timing and Frequency Control," IEEE Transactions on Ultrasonics, Ferroelectrics and Frequency Control, vol. 54, no. 2, pp. 251-270, 2007.

[2] G. Piazza, P. J. Stephanou, and A. P. Pisano, "One and Two Port Piezoelectric Higher Order Contour-Mode MEMS Resonators for Mechanical Signal Processing," Solid-State Electronics, vol. 51, pp. 1596-1608, 2007.

[3] S. Kiaei, S. M. Taleie, and B. Bakkaloglu, "Low-Power High-Q NEMS Receiver Architecture," 2005 IEEE International Symposium on Circuits and Systems (ISCAS 2005), pp. 4401-4404, Kobe, Japan, 2005.

[4] G. Piazza, P. J. Stephanou, and A. P. Pisano, "Piezoelectric Aluminum Nitride Vibrating Contour-Mode MEMS Resonators," Journal of MicroElectroMechanical Systems, vol. 15, no. 6, pp. 1406-1418, 2006.

[5] H. Chandrahalim, D. Weinstein, L. F. Cheow, and S. A. Bhave, "High- $\kappa$ dielectrically transduced MEMS thickness shear mode resonators and tunable channel-select RF filters," Sensors and Actuators A: Physical, vol. 136, no. 2, pp. 527-539, 2007.

[6] C. Zuo, N. Sinha, M. B. Pisani, C. R. Perez, R. Mahameed, and G. Piazza, "Channel-Select RF MEMS Filters Based On Self-Coupled AIN Contour-Mode Piezoelectric Resonators," 2007 IEEE International Ultrasonics Symposium, pp. 1156-1159, New York, USA, 2007.

[7] H.-Y. Tsui and J. Lau, "A 5GHz 56dB Voltage Gain $0.18 \mu \mathrm{m}$ CMOS LNA with Built-in Tunable Channel Filter for Direct Conversion 802.11a Wireless LAN Receiver," 2003 IEEE Radio Frequency Integrated Circuits (RFIC) Symposium, pp. 225-228, Philadelphia, PA, USA, 2003.

[8] G. Piazza, P. J. Stephanou, and A. P. Pisano, "Single-Chip Multiple-Frequency ALN MEMS Filters Based on Contour-Mode Piezoelectric Resonators," Journal of MicroElectroMechanical Systems, vol. 16, no. 2, pp. 319-328, 2007.

[9] N. Sinha, R. Mahameed, C. Zuo, and G. Piazza, "Integration of AlN Micromechanical Contour-Mode Technology Filters with Three-Finger Dual Beam AlN MEMS Switches," 2009 Joint Meeting of the European Frequency and Time Forum and the IEEE International Frequency Control Symposium, pp. 1-4, Besançon, France, 2009.

[10] M. Rinaldi, C. Zuniga, C. Zuo, and G. Piazza, "AlN Contour-Mode Resonators for Narrow-Band Filters above 3 GHz," 2009 Joint Meeting of the European Frequency and Time Forum and the IEEE International Frequency Control Symposium, Besançon, France, 2009.

[11] R. Mahameed, N. Sinha, M. B. Pisani, and G. Piazza, "Dual-Beam Actuation of Piezoelectric AlN RF MEMS Switches Monolithically Integrated with AIN Contour-Mode Resonators," Journal of Micromechanics and Microengineering, vol. 18, no. 10, 2008. 